\title{
Cannabidiol Inhibits Endocannabinoid Signaling in Autaptic Hippocampal Neurons
}

\author{
Alex Straiker, Michaela Dvorakova, Anaelle Zimmowitch, and Ken Mackie \\ Gill Center for Biomolecular Science, Department of Psychological and Brain Sciences, Indiana University, Bloomington, Indiana \\ Received January 23, 2018; accepted April 16, 2018
}

\section{ABSTRACT}

$\Delta^{9}$-Tetrahydrocannabinol (THC) and cannabidiol (CBD) are two main cannabinoid constituents of marijuana and hashish. The pharmacology of $\Delta^{9}$-THC has been extensively studied, whereas our understanding of the pharmacology of CBD has remained limited, despite excitement in CBD's potential role in treating certain pediatric epilepsies and its reputation for attenuating some $\Delta^{9}$-THC-induced effects. It was established early on that CBD binds poorly to the orthosteric site of $\mathrm{CB}_{1}$ or $\mathrm{CB}_{2}$ cannabinoid receptors, and its actions were commonly attributed to other noncannabinoid receptor mechanisms. However, recent evidence suggests that $\mathrm{CBD}$ does indeed act at cannabinoid $\mathrm{CB}_{1}$ receptors as a negative allosteric modulator (NAM) of $\mathrm{CB}_{1}$ signaling. By altering the orthosteric signaling of a G protein-coupled receptor, allosteric modulators greatly increase the richness of $G$ proteincoupled receptor pharmacology. We have recently surveyed candidate $\mathrm{CB}_{1}$ NAMs in autaptic hippocampal neurons, a wellcharacterized neuronal model of endogenous cannabinoid signaling, and have now tested CBD in this model. We find that although CBD has no direct effect on excitatory transmission, it does inhibit two forms of endogenous cannabinoid-mediated retrograde synaptic plasticity: depolarization-induced suppression of excitation and metabotropic suppression of excitation, while not affecting signaling via GABA-B receptors. These results are consistent with the recently described NAM activity of CBD and suggest interesting possible mechanisms for CBD's therapeutic actions.

\section{Introduction}

Although there are many cannabinoids present in marijuana and hashish, two components found in greatest abundance are $\Delta^{9}$-tetrahydrocannabinol (THC) and cannabidiol (CBD) (Elsohly and Slade, 2005). $\Delta^{9}$-THC was identified as the chief psychoactive ingredient of marijuana (Gaoni and Mechoulam, 1964) and has been the subject of thousands of studies in the intervening years. Although isolated earlier (Adams et al., 1940), CBD by contrast has remained a more elusive and poorly studied target, partly because CBD does not compete with conventional orthosteric radioligands for binding at the $\mathrm{CB}_{1}$ cannabinoid receptor (Thomas et al., 1998), but also because CBD itself does not elicit distinctive psychoactive effects (Mechoulam et al., 1970). This picture has gradually changed; varying the ratio of $\Delta^{9}$-THC and CBD in cannabis and cannabinoid preparations has been reported to yield differential effects (Karniol and Carlini, 1973; Hiltunen and Jarbe, 1986; Petitet et al., 1998; Russo and Guy, 2006), and much effort has gone into development of marijuana strains with different proportions of these two components. More recently, CBD has generated considerable interest as an antiepileptic (reviewed in O'Connell et al., 2017) and for other

This work was supported by National Institutes of Health [Grants EY24625, EY24717, DA009158, and DA021696]

https://doi.org/10.1124/mol.118.111864. potential therapeutic properties (reviewed in Maccarrone et al., 2017).

A recent study by Laprairie et al. (2015) revisited the hypothesis that $\mathrm{CBD}$ may interfere with $\mathrm{CB}_{1}$ activation (Petitet et al., 1998) but from the perspective of allosteric modulation. The premise of allosteric modulation is that many receptors have not only a primary orthosteric site of activation, but also one or more allosteric sites. Ligands that bind these sites are termed allosteric ligands and can exert a variety of actions ranging from potentiation/inhibition of the orthosteric signal to agonism/antagonism independent of orthosteric activation. Some major classes of drugs such as the benzodiazepines are well known to act as allosteric modulators, and two allosteric modulators of G proteincoupled receptors, cinacalcet and maraviroc, have been approved for clinical use in the United States. Allosterism at $\mathrm{CB}_{1}$ has seen a flurry of recent interest. A negative allosteric modulator, or negative allosteric modulator (NAM), would bind to $\mathrm{CB}_{1}$ at a secondary site and presumably act either by interfering with the kinetics of orthosteric ligand binding and/or receptor activation/signaling. Such a NAM may lack the side effects seen with orthosteric antagonism (or orthosteric inverse agonism). We have recently surveyed the function of the first-generation NAMs at $\mathrm{CB}_{1}$ using autaptic hippocampal neurons (Straiker et al., 2015). These neurons express $\mathrm{CB}_{1}$ cannabinoid receptors and a form of retrograde signaling known as depolarization-induced suppression of

ABBREVIATIONS: 2-AG, 2-arachidonoyl glycerol; CBD, cannabidiol; Cl, confidence interval; DHPG, dihydrophenylglycine; DSE, depolarizationinduced suppression of excitation; EPSC, excitatory postsynaptic current; MSE, metabotropic suppression of excitation; NAM, negative allosteric modulator; THC, tetrahydrocannabinol. 
excitation (DSE) (Straiker and Mackie, 2005). We have extensively characterized this form of synaptic plasticity, which we find to be 2-arachidonoyl glycerol (2-AG) mediated, and have employed DSE as a tool to explore the pharmacology of endogenous neuronal cannabinoid signaling (e.g., Straiker et al., 2009, 2011a; Jain et al., 2013). We have now tested CBD in this model and report in this work that CBD interferes with endogenous cannabinoid/ $\mathrm{CB}_{1}$-mediated signaling in a concentration-dependent manner and in a fashion consistent with negative allosteric modulation.

\section{Materials and Methods}

Animals and Cell Culture. All animal care and experimental procedures used in this study were approved by the Institutional Animal Care and Use Committee of Indiana University and conform to the National Institutes of Health Guidelines on the Care and Use of Animals. Mouse hippocampal neurons isolated from the CA1-CA3 region were cultured on microislands, as previously described (Furshpan et al., 1976; Bekkers and Stevens, 1991). Briefly, neurons were obtained from animals (at postnatal day 0-2, killed via rapid decapitation without anesthesia) and plated onto a feeder layer of hippocampal astrocytes that had been laid down previously (Levison and McCarthy, 1991). Cultures were grown in highglucose $(20 \mathrm{mM})$ minimum essential media containing $10 \%$ horse serum, without mitotic inhibitors, and used for recordings after 8 days in culture and for no more than 3 hours after removal from culture medium (Straiker and Mackie, 2005). All electrophysiological experiments were performed exclusively on excitatory neurons. All tests were made on neurons from at least two different preparations.

Electrophysiology. When a single neuron is grown on a small island of permissive substrate, it forms synapses-or autapses-onto itself. All experiments were performed on isolated autaptic neurons. Whole-cell, voltage-clamp recordings from autaptic neurons were carried out at room temperature using an Axopatch 200B amplifier (Molecular Devices, Sunnyvale, CA). The extracellular solution contained (millimolars) $\mathrm{NaCl} 119, \mathrm{KCl} 5, \mathrm{CaCl}_{2} 2, \mathrm{MgCl}_{2} 1$, glucose 30, and HEPES 20. Continuous flow of solution through the bath chamber $\left(\sim 2 \mathrm{ml} \times \mathrm{min}^{-1}\right)$ ensured rapid drug application and clearance. Drugs were typically prepared as a stock and then diluted into extracellular solution at their final concentration and used on the same day. Recording pipettes of 1.8-3 $\mathrm{M} \Omega$ were filled with solution containing the following (millimolars): potassium gluconate 121.5, $\mathrm{KCl} 17.5$, $\mathrm{NaCl} 9, \mathrm{MgCl}_{2}$ 1, HEPES 10, EGTA 0.2, MgATP 2, and lithium guanosine triphosphate 0.5. Excitatory postsynaptic currents (EPSCs) were elicited by a brief ( 1 milliseconds) depolarization to $0 \mathrm{mV}$. DSE was elicited by longer depolarizations (50 milliseconds10 seconds) also to $0 \mathrm{mV}$. Access resistance was monitored, and only cells with a stable access resistance were included for data analysis.

Materials. Baclofen was purchased from Sigma-Aldrich (St. Louis, MO). CBD was obtained from National Institute on Drug Abuse Drug Supply Program (Research Triangle Park, NC). CBD was stored at $-80^{\circ} \mathrm{C}$ and diluted shortly before use.

Statistical Analysis. For electrophysiology analyses, depolarization response curves were obtained to determine inhibition of excitatory synaptic transmission by endogenous 2-AG by depolarizing neurons for progressively longer durations $(50,100,300$, and 500 milliseconds; 1,3 , and 10 seconds). The data were fitted with a nonlinear regression (Sigmoidal dose response; GraphPad Prism 6, La Jolla, CA), allowing calculation of an $\mathrm{ED}_{50}$, the effective dose or duration of depolarization at which a $50 \%$ inhibition is achieved. Statistically significant differences in these curves were taken as nonoverlapping $95 \%$ confidence intervals (CIs). Values on graphs are presented \pm S.E.M.

\section{Results}

CBD Suppresses CB $_{1}$ Receptor-Mediated DSE. We tested the ability of CBD to suppress synaptic transmission in autaptic hippocampal neurons, first recording from cells to obtain baseline EPSCs, and then treating cells for 5 minutes with CBD (500 nM). As shown in Fig. 1A, CBD did not inhibit EPSCs, indicating that at $500 \mathrm{nM}$ CBD does not directly inhibit (or activate) neurotransmission in these neurons [Fig. 1A; relative EPSC charge (500 nM CBD): $1.03 \pm 0.03, n=5$ ].

Depolarization of excitatory autaptic hippocampal neurons elicits a form of endocannabinoid (2-AG) and CB1-mediated retrograde inhibition termed DSE (Straiker and Mackie, 2005). This can be quantified by subjecting the neuron to a series of successively longer depolarizations (50, 100, 300, and 500 milliseconds; 1,3 , and 10 seconds), resulting in progressively greater inhibition of neurotransmission (Straiker et al., 2011b). A sample time course from such a depolarization series is shown in Fig. 1D. Plotting maximal inhibition as a result of each duration of depolarization yields a depolarization-response curve that permits the characterization of some pharmacological properties of endocannabinoid signaling, including the calculation of an $\mathrm{ED}_{50}$ (depolarization). This $\mathrm{ED}_{50}$ corresponds to the duration of depolarization that results in $50 \%$ of the maximal inhibition. A NAM would be expected to shift a depolarization-response curve up and to the right (i.e., less DSE in a noncompetitive fashion). We tested CBD at 100 and $500 \mathrm{nM}$ and $2 \mu \mathrm{M}$, finding that $500 \mathrm{nM}$ and $2 \mu \mathrm{M}$ CBD substantially shifted the $\mathrm{ED}_{50}$ [Fig. 1, B and C; baseline $\mathrm{ED}_{50}$ (duration of depolarization [95\% CI]): 1.0 second (0.6-1.5); $\mathrm{ED}_{50}$ (100 nM CBD): 1.6 seconds (1.1-2.2); $n=7$; $\mathrm{ED}_{50}(500 \mathrm{nM} \mathrm{CBD}): 4.9$ seconds $(2.4-10.2) ; n=5 ; \mathrm{ED}_{50}(2 \mu \mathrm{M}$ CBD): 2.8 seconds (1.6-4.8); $n=5 ; 95 \%$ CI nonoverlapping for $500 \mathrm{nM}$ and $2 \mu \mathrm{M}$ CBD versus baseline]. Maximal inhibition was significantly decreased after treatment with either $500 \mathrm{nM}$ or $2 \mu \mathrm{M}$ CBD [baseline Emax (relative EPSC charge [95\% CI]): 0.37 (0.28-0.47); Emax in presence of $100 \mathrm{nM} \mathrm{CBD:}$ 0.52 (0.47-0.58); 500 nM CBD: 0.67 (0.56-0.78); $2 \mu \mathrm{M}$ CBD: 0.77 (0.71-0.82); 95\% CI nonoverlapping for $500 \mathrm{nM}$ and $2 \mu \mathrm{M}$ versus baseline]. Averaged time courses of DSE in response to a 3-second depolarization before and after treatment with $500 \mathrm{nM}$ CBD are shown in Fig. 1E. We additionally tested whether CBD would interfere with exogenously applied 2-AG to confirm that the effect of CBD was not due to a postsynaptic alteration of 2-AG synthesis and/or release. We used a halfmaximal concentration of $2-\mathrm{AG}, 500 \mathrm{nM}$, and found that $500 \mathrm{nM}$ CBD diminished EPSC inhibition by 2-AG on reapplication in the same cells [Fig. 1, F and G; relative EPSC charge after $500 \mathrm{nM} 2$-AG: $0.63 \pm 0.03$; relative charge with 2-AG and CBD (both $500 \mathrm{nM}$ ): $0.81 \pm 0.04, n=7 ; P<0.05$ paired $t$ test].

If the effect of CBD was due to a general inhibition of presynaptic $\mathrm{G}$ protein-coupled receptor signaling, then one would expect CBD to also suppress the activity of other $\mathrm{G}_{\mathrm{i} / \mathrm{o}^{-}}$ coupled receptors. GABA-B receptors also presynaptically inhibit neurotransmitter release in autaptic hippocampal neurons (Straiker et al., 2002). We found that inhibition of neurotransmitter release by GABA-B activation with baclofen $(25 \mu \mathrm{M})$ remained intact in the presence of $500 \mathrm{nM}$ CBD [Fig. $1 \mathrm{H}$; relative EPSC charge after $25 \mu \mathrm{M}$ baclofen ( \pm S.E.M.): $0.30 \pm$ 0.07; baclofen + CBD (500 nM): $0.26 \pm 0.04 ; n=5 ; P>0.05$, paired $t$ test]. 

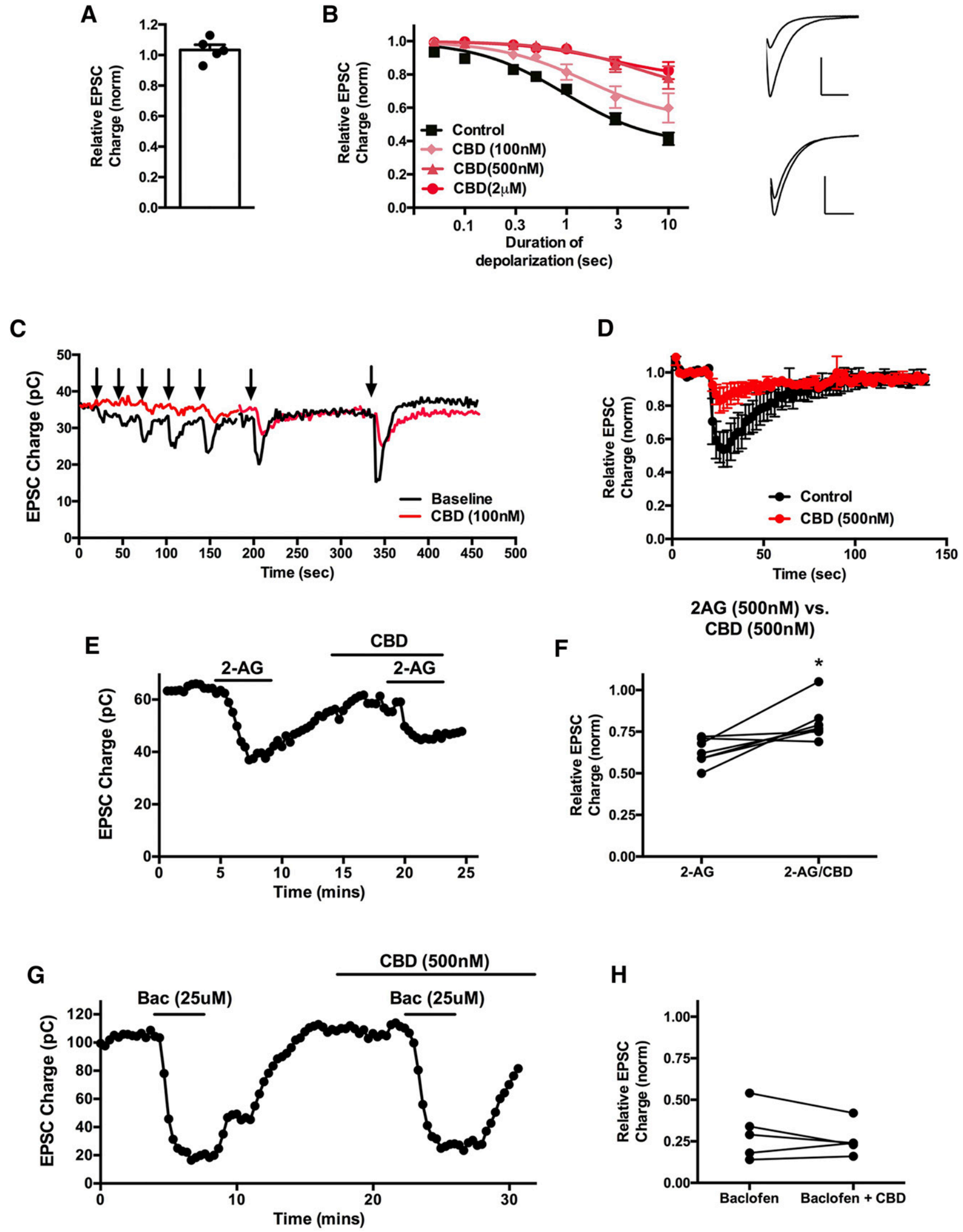

Fig. 1. CBD suppresses $\mathrm{CB}_{1}$ receptor-mediated DSE. (A) $\mathrm{CBD}(500 \mathrm{nM})$ does not directly inhibit excitatory postsynaptic currents. (B) The 100 or $500 \mathrm{nM}$ CBD treatment (5 minutes) shifts the response curve for DSE in a concentration-dependent manner. Inset shows sample EPSCs before and after DSE under baseline conditions (top) and after CBD (500 nM) treatment (bottom). (C) Sample time course showing DSE responses to progressively longer depolarizations before and after $100 \mathrm{nM}$ CBD treatment. (D) Averaged DSE time course in response to 3-second depolarization before and after $500 \mathrm{nM}$ CBD treatment. (E) Representative experiment showing that half-maximal 2-AG responses $(500 \mathrm{nM})$ are diminished by $500 \mathrm{nM}$ CBD. (F) Inhibition by 2 -AG (500 $\mathrm{nM})$ alone gives a stronger response 
A
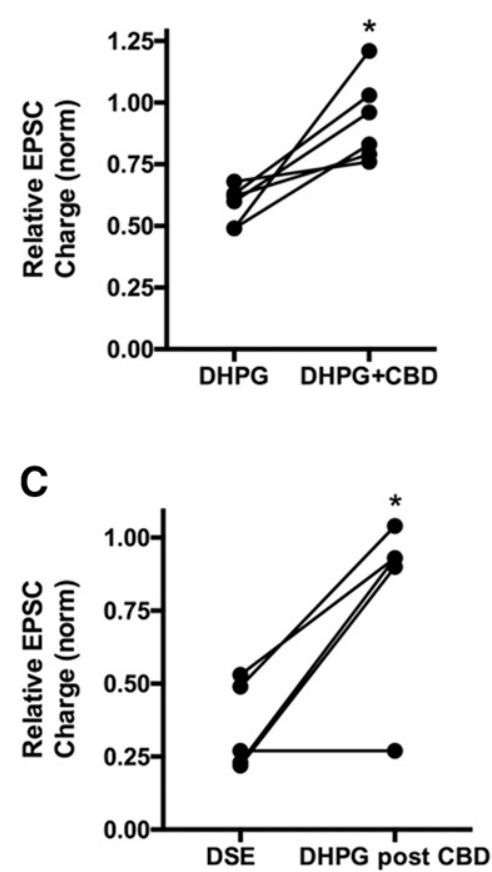

B
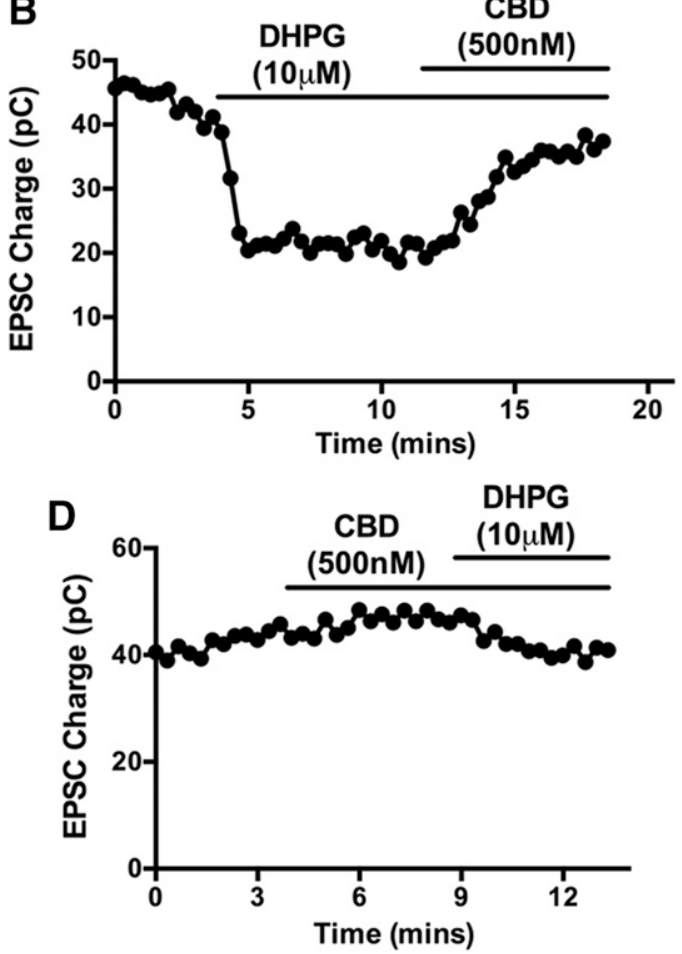

Fig. 2. CBD suppresses endogenous $\mathrm{CB}_{1}$ receptor-mediated metabotropic suppression of excitation. (A) mGluR group I agonist DHPG $(10 \mu \mathrm{M})$ suppresses EPSCs, but this is reversed by treatment with $500 \mathrm{nM}$ CBD. (B) Sample time course showing DHPG response reversed by subsequent cotreatment with CBD. (C) Bar graph shows maximal inhibition due to DSE before CBD and the effect of DHPG $(10 \mu \mathrm{M})$ when applied after/with CBD (500 nM). (D) Sample time course showing CBD treatment, followed by coapplication of CBD and DHPG. $* P<0.05$ by paired $t$ test.
CBD Also Suppresses CB1 Receptor-Mediated Metabotropic Suppression of Excitation. We additionally tested whether CBD has a similar effect on a related form of cannabinoid-mediated retrograde synaptic plasticity known as metabotropic suppression of excitation (MSE). First described in the cerebellum (Maejima et al., 2001), we have characterized MSE in autaptic hippocampal neurons and shown it to be CB1 receptor dependent (Straiker and Mackie, 2007). We have additionally shown that MSE (like DSE) involves 2-AG rather than anandamide because $2-\mathrm{AG}$, but not anandamide, fits the kinetic profile of MSE and because inhibition or knockdown of diacylglycerol lipases eliminates MSE (Jain et al., 2013).

Briefly, postsynaptic $\mathrm{G}_{\mathrm{q}}$-coupled receptors have been found to activate a signaling cascade, involving $\mathrm{G}_{\mathrm{q}}$, phospholipase $\mathrm{C}$, and diacylglycerol lipase, which results in the production of 2-AG that, like the 2-AG, produced as a result of DSE, retrogradely crosses the synapse, where it activates presynaptic CB1 receptors and thereby inhibits neurotransmission (reviewed in Kano et al., 2009). In addition to metabotropic mGluR group I receptors (Maejima et al., 2001), several other $\mathrm{G}_{\mathrm{q}}$-coupled receptors have been found capable of inducing MSE, including muscarinic (Kim et al., 2002), orexin (HajDahmane and Shen, 2005), and substance P (Drew et al., 2009) receptors. We first treated neurons with the mGluR group I agonist dihydroxyphenylglycine (DHPG) $(10 \mu \mathrm{M})$ to transient MSE, then coapplied DHPG with CBD (500 nM), finding that this substantially reversed DHPG-induced MSE (Fig. 2, A and B; inhibition with $10 \mu \mathrm{M}$ DHPG: $0.59 \pm 0.07$; DHPG + CBD (500 nM): $0.93 \pm 0.16, n=6, P<0.05$, paired $t$ test).
Similarly, application of CBD before DHPG prevented the full effect of DHPG-induced MSE [Fig. 2, C and D; inhibition with $10 \mu \mathrm{M}$ DHPG in presence of CBD (500 nM): $0.81 \pm 0.14$, $n=5$ ]. For these experiments, responses are compared with DSE in the same cells elicited before treatment with either drug (Fig. 2C; DSE inhibition: $0.36 \pm 0.07, n=5, P<0.05$ paired $t$ test versus DHPG $+\mathrm{CBD}$ ). DSE and MSE induce the same degree of CB1-dependent inhibition in a given cell (Straiker and Mackie, 2007).

\section{Discussion}

We have tested the important nonpsychoactive component of marijuana, CBD, in a well-characterized model of endogenous cannabinoid signaling. We find that whereas CBD does not directly modulate neurotransmission in this model, it does suppress two forms of endogenous cannabinoid-mediated synaptic plasticity, while not affecting inhibition of synaptic transmission by GABA-B receptors. This finding extends a recent report that $\mathrm{CBD}$ acts as a NAM at $\mathrm{CB}_{1}$ receptors (Laprairie et al., 2015) to models of endocannabinoid-mediated synaptic plasticity.

In considering the role and therapeutic potential of cannabinoids, for many years $\Delta^{9}$-THC has received the greatest attention. As the chief psychoactive ingredient of cannabis and as an established (albeit low efficacy) agonist at both of the canonical cannabinoid receptors, THC has been a natural object of study. The pharmacology of CBD, in contrast, has been difficult to sort out. CBD competes poorly with 
cannabinoid receptor ligand binding to cannabinoid receptors (Thomas et al., 1998), leading to an initial (and persistent) conclusion that any action of CBD necessarily occurs through noncannabinoid receptors. It may seem surprising that CBD and THC are structurally similar (and with the same molecular weight) yet exhibit different orthosteric binding to CB1 receptors, but this may be accounted for by steric differences between these molecules as explored in structure-activity studies (e.g., Papahatjis et al., 2002). However, it subsequently became clear that CBD does interfere with cannabinoid function at a potency greater than that expected based on the earlier orthosteric binding data (Thomas et al., 2007). This raised the possibility that CBD acts noncompetitively relative to the orthosteric site at cannabinoid receptors, although this still left open numerous potential mechanisms of action, including action via unrelated receptors or signaling pathways. The recent finding that $\mathrm{CBD}$ has a profile consistent with potent negative allosteric modulation of $\mathrm{CB}_{1}$ signaling stimulated by 2-AG and THC in a broad range of signaling assays (Laprairie et al., 2015) therefore offered a significant and welcome insight. However, awareness of this study and its implications for CBD function are still limited, and CBD is still commonly assumed by many to be inactive at $\mathrm{CB}_{1}$ receptors (e.g., Devinsky et al., 2017).

Cannabinoid $\mathrm{CB}_{1}$ receptors are abundant and widely distributed in the central nervous system (Herkenham et al., 1990). In $\mathrm{CB}_{1}$-expressing neurons, they are highly enriched on presynaptic terminals and have been shown to mediate several forms of neuronal plasticity (reviewed in Kano et al., 2009), among them DSE (Kreitzer and Regehr, 2001), the related depolarization-induced suppression of inhibition (Wilson and Nicoll, 2001), and metabotropic suppression of excitation (MSE) (Maejima et al., 2001). It is therefore important to determine in a well-characterized and tractable model the extent to which $\mathrm{CBD}$ alters $\mathrm{CB}_{1}$-mediated plasticity. Excitatory autaptic hippocampal neurons express DSE and MSE in an architecturally simple system consisting of a single neuron synapsing onto itself, an arrangement free of network-signaling complications that arise in more elaborate preparations. Moreover, the model can be said to be well characterized insofar as it has been used for studies of cannabinoid signaling in $\sim 25$ publications to date. Our observations clearly establish that CBD inhibits two forms of endocannabinoid-mediated synaptic plasticity. However, whereas our findings buttress and extend those of Laprairie et al. (2015), the experiments were designed to qualitatively demonstrate antagonism, not allosterism per se. The autaptic neuronal model is unsuited to Schild analyses, which require steady state receptor activation, due to the transient nature of 2-AG engagement of the $\mathrm{CB}_{1}$ receptor following its release. Thus, the strongest conclusion of our studies is that CBD noncompetitively inhibits $\mathrm{CB} 1$ receptor activation by $2-\mathrm{AG}$ in a model of endogenous cannabinoid signaling, consistent with negative allosteric modulation.

In principle, CBD might be acting at other components of the machinery that regulates eCB synthesis, transport, or metabolism. Our experiments used bath-applied 2-AG argue against an effect on the 2-AG synthesizing or release machinery. On the presynaptic side, we have shown that the time course of DSE is determined by monoacylglycerol lipase metabolism (Straiker et al., 2009) and that fatty acid amide hydrolase only plays a role when overexpressed (Straiker et al., 2011a). Diminishment of DSE responses via a presynaptic mechanism would require enhanced 2-AG metabolism or transport, which would be seen not as diminished maximal inhibition, but rather as a more rapid DSE decay. Indeed, we have previously shown that the more rapid depolarization-induced suppression of inhibition time course in autaptic neurons is a function of more rapid metabolism by a combination of monoacylglycerol lipase and cyclooxygenase 2 (Straiker and Mackie, 2009). CBD is therefore unlikely to be acting on the synthetic, transport, or metabolizing machinery for 2-AG in this system.

Having said this, does the observed antagonism occur at relevant concentrations? Do the concentrations of CBD in the body after marijuana use or likely therapeutic treatments approach a level at which they could be expected to antagonize $\mathrm{CB}_{1}$ receptor signaling, as we have seen in this work? In a related question, is negative allosteric modulation of $\mathrm{CB}_{1}$ by $\mathrm{CBD}$ a bug or a feature? Is $\mathrm{CB}_{1}$ antagonism the basis for physiologic consequences that have been noted for CBD treatment? Several ongoing clinical trials are evaluating CBD for epilepsy treatment, particularly for forms of childhood epilepsy, including Dravet's Syndrome and LennoxGastaut Syndrome (O'Connell et al., 2017). A recent clinical trial testing CBD at $20 \mathrm{mg} / \mathrm{kg}$ per day for several weeks found that CBD reduced tonic/clonic seizures in a significant fraction of children with Dravet's Syndrome (Devinsky et al., 2017, 2018). The same group determined that $20 \mathrm{mg} / \mathrm{kg}$ daily treatment yields peak plasma levels of $\sim 1 \mu \mathrm{M}$ after 22 days of treatment, i.e., above the concentrations tested in this study (100, $500 \mathrm{nM})$ that substantially inhibit endocannabinoid signaling (Devinsky et al., 2018). Plasma levels of CBD obtained during recreational consumption are more difficult to quantify because of the considerable variety of strains, formulations, and modes of consumption. Over the past 20 years, most marijuana consumption involved low-CBD strains, but several high-CBD strains have been developed. A hypothetical cigarette containing $0.75 \mathrm{~g}$ marijuana, with $5 \%$ CBD, would contain $30 \mathrm{mg}$ CBD. A study involving the smoking of a cigarette containing $19 \mathrm{mg}$ CBD was found to result in blood plasma levels of $110 \mathrm{ng} / \mathrm{ml}(\sim 340 \mathrm{nM})($ Ohlsson et al., 1986) at 3 minutes post-treatment, but declined to $10 \mathrm{ng} / \mathrm{ml}$ at 1 hour. A smoker might therefore experience a brief NAM effect from smoking, which likely corresponds to the time of maximal THC brain levels. Interestingly, whereas simultaneous inhalation of THC and CBD attenuates some aspects of THC psychoactivity, sequential (separated by 30 minutes) inhalation of CBD and then THC did not (Dalton et al., 1976). CBD varieties with still higher concentrations of CBD, in excess of $15 \%$, have been developed [e.g., Charlotte's Web (Maa and Figi, 2014)]. The same hypothetical cigarette containing this high CBD cannabis would presumably transiently increase CBD in excess of $1 \mu \mathrm{M}$ plasma. However, the effect would likely last only a few minutes. Studies of oral consumption of CBD in doses likely encountered in recreational cannabis use found relatively low CBD plasma concentrations. For instance, Agurell et al. (1981) reported that $40 \mathrm{mg}$ CBD ingested in cookies yielded only an average of $5.5 \mathrm{ng} / \mathrm{ml}(\sim 20 \mathrm{nM})$ of CBD in plasma.

Cannabis containing substantial CBD has been reported to yield fewer adverse psychologic side effects than THC alone, raising the question of whether a component of cannabis such as CBD might actively moderate the effects of THC (reviewed 
in McPartland et al., 2015). The inhibition of the $\mathrm{CB}_{1}$ signaling we have observed in this study offers one potential mechanism for such an attenuation of selected THC effects. A related question is whether negative allosteric modulation of $\mathrm{CB}_{1}$ by CBD might contribute to its proposed antiepileptic properties, or alternatively contribute to the reported side effects? The identification of CBD as a NAM is recent enough that this remains a largely unexplored question.

In summary, we find that CBD antagonizes two forms of endogenous cannabinoid $\mathrm{CB}_{1}$ receptor-mediated synaptic plasticity in autaptic hippocampal neurons. The inhibition profile of $\mathrm{CBD}$ is consistent with the recently reported negative allosteric modulation at $\mathrm{CB}_{1}$ receptors and occurs at or below concentrations that are likely to be encountered in the body in the course of therapeutic CBD consumption.

\section{Authorship Contributions}

Participated in research design: Straiker, Mackie.

Conducted experiments: Straiker, Dvorakova, Zimmowitch.

Performed data analysis: Straiker, Dvorakova.

Wrote or contributed to the writing of the manuscript: Straiker, Mackie.

\section{References}

Adams R, Hunt M, and Clark JH (1940) Structure of cannabidiol, a product isolated from the marihuana extract of Minnesota wild hemp. J Am Chem Soc 62 196-200.

Agurell S, Carlsson S, Lindgren JE, Ohlsson A, Gillespie H, and Hollister L (1981) Interactions of delta 1-tetrahydrocannabinol with cannabinol and cannabidiol following oral administration in man: assay of cannabinol and cannabidiol by mass fragmentography. Experientia 37:1090-1092.

Bekkers JM and Stevens CF (1991) Excitatory and inhibitory autaptic currents in isolated hippocampal neurons maintained in cell culture. Proc Natl Acad Sci USA 88:7834-7838.

Dalton WS, Martz R, Lemberger L, Rodda BE, and Forney RB (1976) Influence of cannabidiol on delta-9-tetrahydrocannabinol effects. Clin Pharmacol Ther 19: 300-309.

Devinsky O, Cross JH, Laux L, Marsh E, Miller I, Nabbout R, Scheffer IE, Thiele EA and Wright S; Cannabidiol in Dravet Syndrome Study Group (2017) Trial of cannabidiol for drug-resistant seizures in the Dravet syndrome. $N$ Engl J Med 376: 2011-2020.

Devinsky O, Patel AD, Thiele EA, Wong MH, Appleton R, Harden CL, Greenwood S, Morrison G, and Sommerville K; GWPCARE1 Part A Study Group (2018) Randomized, dose-ranging safety trial of cannabidiol in Dravet syndrome. Neurology 90:e1204-e1211.

Drew GM, Lau BK, and Vaughan CW (2009) Substance P drives endocannabinoidmediated disinhibition in a midbrain descending analgesic pathway. J Neurosci $\mathbf{2 9}$ $7220-7229$.

Elsohly MA and Slade D (2005) Chemical constituents of marijuana: the complex mixture of natural cannabinoids. Life Sci 78:539-548.

Furshpan EJ, MacLeish PR, O'Lague PH, and Potter DD (1976) Chemical transmission between rat sympathetic neurons and cardiac myocytes developing in microcultures: evidence for cholinergic, adrenergic, and dual-function neurons. Proc Natl Acad Sci USA 73:4225-4229.

Gaoni Y and Mechoulam R (1964) Isolation, structure and partial synthesis of an active constituent of hashish. J Am Chem Soc 86:1646-1647.

Haj-Dahmane S and Shen RY (2005) The wake-promoting peptide orexin-B inhibits glutamatergic transmission to dorsal raphe nucleus serotonin neurons through retrograde endocannabinoid signaling. J Neurosci 25:896-905

Herkenham M, Lynn AB, Little MD, Johnson MR, Melvin LS, de Costa BR, and Rice KC (1990) Cannabinoid receptor localization in brain. Proc Natl Acad Sci USA 87: $1932-1936$

Hiltunen AJ and Järbe TU (1986) Cannabidiol attenuates delta 9-tetrahydrocannabinollike discriminative stimulus effects of cannabinol. Eur J Pharmacol 125:301-304.

Jain T, Wager-Miller J, Mackie K, and Straiker A (2013) Diacylglycerol lipaseo (DAGL $\alpha$ ) and DAGL $\beta$ cooperatively regulate the production of 2-arachidonoyl glycerol in autaptic hippocampal neurons. Mol Pharmacol 84:296-302.
Kano M, Ohno-Shosaku T, Hashimotodani Y, Uchigashima M, and Watanabe M (2009) Endocannabinoid-mediated control of synaptic transmission. Physiol Rev 89:309-380.

Karniol IG and Carlini EA (1973) Pharmacological interaction between cannabidiol and delta 9-tetrahydrocannabinol. Psychopharmacology (Berl) 33:53-70.

Kim J, Isokawa M, Ledent C, and Alger BE (2002) Activation of muscarinic acetylcholine receptors enhances the release of endogenous cannabinoids in the hippocampus. J Neurosci 22:10182-10191.

Kreitzer AC and Regehr WG (2001) Retrograde inhibition of presynaptic calcium influx by endogenous cannabinoids at excitatory synapses onto Purkinje cells. Neuron 29:717-727.

Laprairie RB, Bagher AM, Kelly ME, and Denovan-Wright EM (2015) Cannabidiol is a negative allosteric modulator of the cannabinoid CB1 receptor. $\mathrm{Br} J$ Pharmacol 172:4790-4805.

Levison SW and McCarthy KD (1991) Characterization and partial purification of AIM: a plasma protein that induces rat cerebral type 2 astroglia from bipotential glial progenitors. $J$ Neurochem 57:782-794.

Maa E and Figi P (2014) The case for medical marijuana in epilepsy. Epilepsia 55: $783-786$.

Maccarrone M, Maldonado R, Casas M, Henze T, and Centonze D (2017) Cannabinoids therapeutic use: what is our current understanding following the in troduction of THC, THC:CBD oromucosal spray and others? Expert Rev Clin Pharmacol 10:443-455.

Maejima T, Hashimoto K, Yoshida T, Aiba A, and Kano M (2001) Presynaptic inhibition caused by retrograde signal from metabotropic glutamate to cannabinoid receptors. Neuron 31:463-475.

McPartland JM, Duncan M, Di Marzo V, and Pertwee RG (2015) Are cannabidiol and $\Delta(9)$-tetrahydrocannabivarin negative modulators of the endocannabinoid system? A systematic review. Br J Pharmacol 172:737-753.

Mechoulam R, Shani A, Edery H, and Grunfeld Y (1970) Chemical basis of hashish activity. Science 169:611-612.

O'Connell BK, Gloss D, and Devinsky O (2017) Cannabinoids in treatment-resistant epilepsy: a review. Epilepsy Behav 70:341-348.

Ohlsson A, Lindgren JE, Andersson S, Agurell S, Gillespie H, and Hollister LE (1986) Single-dose kinetics of deuterium-labelled cannabidiol in man after smoking and intravenous administration. Biomed Environ Mass Spectrom 13:77-83.

Papahatjis DP, Nikas SP, Andreou T, and Makriyannis A (2002) Novel $1^{\prime}, 1^{\prime}$-chain substituted Delta(8)-tetrahydrocannabinols. Bioorg Med Chem Lett 12:3583-3586.

Petitet F, Jeantaud B, Reibaud M, Imperato A, and Dubroeucq MC (1998) Complex pharmacology of natural cannabinoids: evidence for partial agonist activity of delta9-tetrahydrocannabinol and antagonist activity of cannabidiol on rat brain cannabinoid receptors. Life Sci 63:PL1-PL6.

Russo E and Guy GW (2006) A tale of two cannabinoids: the therapeutic rationale for combining tetrahydrocannabinol and cannabidiol. Med Hypotheses 66:234-246.

Straiker A, Hu SS, Long JZ, Arnold A, Wager-Miller J, Cravatt BF, and Mackie K (2009) Monoacylglycerol lipase limits the duration of endocannabinoid-mediated depolarization-induced suppression of excitation in autaptic hippocampal neurons. Mol Pharmacol 76:1220-1227.

Straiker A and Mackie K (2005) Depolarization-induced suppression of excitation in murine autaptic hippocampal neurones. J Physiol 569:501-517.

Straiker A and Mackie K (2007) Metabotropic suppression of excitation in murine autaptic hippocampal neurons. J Physiol 578:773-785.

Straiker A and Mackie K (2009) Cannabinoid signaling in inhibitory autaptic hippocampal neurons. Neuroscience 163:190-201.

Straiker A, Mitjavila J, Yin D, Gibson A, and Mackie K (2015) Aiming for allosterism: evaluation of allosteric modulators of CB1 in a neuronal model. Pharmacol Res 99:370-376.

Straiker A, Wager-Miller J, Hu SS, Blankman JL, Cravatt BF, and Mackie K (2011a) COX-2 and fatty acid amide hydrolase can regulate the time course of depolarization-induced suppression of excitation. Br J Pharmacol 164:1672-1683.

Straiker A, Wager-Miller J, Hutchens J, and Mackie K (2011b) Differential signalling in human cannabinoid CB1 receptors and their splice variants in autaptic hippocampal neurones. Br J Pharmacol 165:2660-2671.

Straiker AJ, Borden CR, and Sullivan JM (2002) G-protein alpha subunit isoforms couple differentially to receptors that mediate presynaptic inhibition at rat hippocampal synapses. $J$ Neurosci 22:2460-2468.

Thomas A, Baillie GL, Phillips AM, Razdan RK, Ross RA, and Pertwee RG (2007) Cannabidiol displays unexpectedly high potency as an antagonist of CB1 and CB2 receptor agonists in vitro. Br J Pharmacol 150:613-623.

Thomas BF, Gilliam AF, Burch DF, Roche MJ, and Seltzman HH (1998) Comparative receptor binding analyses of cannabinoid agonists and antagonists. J Pharmacol Exp Ther 285:285-292.

Wilson RI and Nicoll RA (2001) Endogenous cannabinoids mediate retrograde signalling at hippocampal synapses. Nature 410:588-592.

Address correspondence to: Dr. Alex Straiker, Indiana University, 1101 East 10th Street, Bloomington, IN 47405. E-mail: straiker@indiana.edu 